\title{
Welcome to the Special Section on IDW '15
}

Editor-in-Chief Keiichi Betsui

It is a great pleasure to introduce the special section " IDW '15 " in the Transactions on Media Technology and Applications (MTA). The objective of the special section is focus on the advances in display materials, devices, and systems. Initially, we called for papers in the following areas:

- Based on the presentation in IDW '15

- Based on the display materials, devices, and systems

in the fields covered by IDW '15

With the great effort made by the associate editors in the guest editorial committee and anonymous reviewers, we have selected five high quality papers (We published three English papers in MTA and published two papers of Japanese in ITEJ). We are very grateful to all the authors who have submitted their excellent works to this issue and all the guest editors and reviewers. We hope that this special section will promote research in boundary fields.

MTA Editorial Committee members list (Vol. 4, No. 4, 2016)

Editor-in-Chief

Keiichi Betsui

HITACHI

\section{Associate Editor-in-Chief}

Yoshihide Fujisaki NHK

\section{$\underline{\text { Associate Editors }}$}

Kentaro Okuyama

Shigeto Yoshida

Masahiro Baba

Hiroyuki Yamakita
JDI

SHARP

TOSHIBA

JOLED
Yoshiki Nakajima

Atsushi Nakamura

Takahiro Ishinabe

Masahiro Yamaguchi
NHK

Shizuoka University

Tohoku University

Tokyo Institute of Technology 